\title{
THE IDEAL PROFILE (INCREASE IN B-LINES/DELTA B-LINES) ON LUNG ULTRASOUND FOR DIAGNOSING WEANING INDUCED PULMONARY EDEMA IN VENTILATED PATIENTS
}

\author{
Ghulam Rasheed, Zahid Siddique Shad*, Tooba Mehreen**, Nusrat Kharadi**, Moazma Ramzan**, Azmat Abdullah**, Kiran Abbas*** \\ Tabba Heart Institute, Karachi Pakistan, *Indus Hospital, Muzaffargarh Pakistan, **Shifa International Hospital, Islamabad Pakistan, *** Jinnah Post Graduate \\ Medical Center, Karachi Pakistan
}

\section{ABSTRACT}

Objective: To ascertain the ideal number of B-lines on lung ultrasound for the diagnosis of weaning induced pulmonary edema in ventilated patients.

Study Design: Prospective observational study.

Place and Duration of Study: Department of Medicine, Shifa International Hospital, Islamabad, from Jan to Aug 2020.

Methodology: All the patients over the age of 18 years who were on mechanical ventilation in a medical intensive care unit were included in the study. The patients were given spontaneous breathing trials as a protocol for weaning from mechanical ventilation. Lung ultrasound was performed on 4 points of anterior chest wall before and after spontaneous breathing trials. Before and after spontaneous breathing trials counting of B lines was done on ultrasound of lung and comparison of increase in B lines (Delta-B-lines) was done with reference diagnosis of weaning induced pulmonary edema diagnosed by intensivist who was blinded to the results of lung ultrasound.

Results: The study included 42 patients including $23(54.8 \%)$ men and $19(45.2 \%)$ women. 14 cases failed spontaneous breathing trials. Seven cases $(16.7 \%)$ had weaning induced pulmonary edema. Delta-B-lines $\geq 6$ diagnosed weaning induced pulmonary edema with $100 \%$ accuracy. Out of the remaining seven patients with weaning failure but without weaning induced pulmonary edema, $6(28.6 \%)$ had Delta-B-lines $\geq 6$. The ultrasound lung technique had a $100 \%$ sensitivity profile to detect weaning induced pulmonary edema and a specificity of $77.78 \%$.

Conclusion: The study indicates that Delta-B-lines $\geq 6$ diagnosed the weaning induced pulmonary edema with the best accuracy.

Keywords: Heart failure, Ideal profile, Mechanical ventilation, Spontaneous breathing trials, Weaning induced pulmonary edema.

How to Cite This Article: Rasheed G, Shad ZS, Mehreen T, Kharadi N, Ramzan M, Abdullah A, Abbas K. The Ideal Profile (Increase in B-Lines/Delta BLines) on Lung Ultrasound for Diagnosing Weaning Induced Pulmonary Edema in Ventilated Patients. Pak Armed Forces Med J 2021; 71(6): 2220-2223. Doi: https://doi.org/10.51253/pafmj.v71i6.5691

This is an Open Access article distributed under the terms of the Creative Commons Attribution License (https://creativecommons.org/licenses/by-nc/4.0/), which permits unrestricted use, distribution, and reproduction in any medium, provided the original work is properly cited.

\section{INTRODUCTION}

Critically ill patients who require invasive ventilation may suffer from spontaneous breathing trial (SBT) failure upon weaning from mechanical ventilation. ${ }^{1}$ The etiology of weaning failure is not very well understood however, patients with pre-existing conditions such as diabetes mellitus, chronic obstructive pulmonary disease (COPD), cardiac dysfunction or hypoxemia are associated with higher risk of weaning failure during extubation. ${ }^{1,2}$ In critically ill patients on mechanical ventilation, weaning failure is an independent predictor of poor outcome. ${ }^{3}$ Determining the reasons for weaning failure are important to devise appropriate treatment and make weaning more successful.

During SBT, cardio-pulmonary interactions may lead to an imbalance between cardiac preload and afterload subsequently causing pulmonary edema known as weaning induced pulmonary edema (WIPO). ${ }^{4} \mathrm{WIPO}$

Correspondence: Dr Ghulam Rasheed, Fellow Critical Care Medicine, Shifa International Hospital, Islamabad Pakistan Pakistan

Received: 16 Nov 2020; revision received: 21 Dec 2020; accepted: 21 Dec 2020 is one of the most known treatable causes of weaning failure, which is why the detection of WIPO is of paramount significance. ${ }^{5}$

There are different invasive and noninvasive methods of diagnosing WIPO. Pulmonary artery wedge pressure measured during an SBT provides the direct evidence of rise in hydrostatic pulmonary pressure. ${ }^{6}$ Apart from the measurement of pulmonary artery occlusion pressure, less invasive methods for detecting pulmonary edema have been established. ${ }^{7}$ The indirect methods include evaluation of blood volume, rise in B-type natriuretic peptide (BNP) level, elevation in the filling pressure of the left ventricle at transthoracic echocardiography (TTE) or expansion in lung water in extravascular space. ${ }^{8}$ These less invasive diagnostic approaches are more appealing to the intensivist as they are less time consuming and promptly establish the diagnosis of WIPO. ${ }^{6}$ Lung ultrasound is one of the least invasive and most time effective methods to establish the diagnosis of WIPO in patients with failed weaning after invasive mechanical ventilation. ${ }^{7}$ 
Lung ultrasound can establish the diagnosis of WIPO by detecting multiple B-lines on imaging indicating interstitial syndrome. Many researchers acknowledge the role of lung ultrasonography in the detection of pulmonary edema of hemodynamic origin. ${ }^{8,9} \mathrm{Num}-$ ber of B-lines has been correlated with N-terminal-proBNP (NT-pro-BNP) in patients with shortness of breath and with accumulation of water in extravascular spaces of lungs. ${ }^{10}$

Although lung ultrasonography has never been evaluated as the solitary diagnostic modality to detect WIPO, however, considering the advantages of a noninvasive technique over invasive diagnostic tools, it makes sense to determine the effectiveness of using lung ultrasonography in this regard. Hence, the current study evaluated the use of lung ultrasonography in detecting any changes in the frequency of B-lines (Delta-B-lines) as an indirect method of diagnosing WIPO. The purpose of this study was to determine the ideal change in number of B-line (Delta-B-lines) that could reliably establish the diagnosis of WIPO in patients who had SBT failure at the time of extubation.

\section{METHODOLOGY}

A prospective observational study was carried out at the department of Critical Care Medicine at Shifa International Hospital Islamabad, from January to August 2020. The study was approved by the ethical review board of Shifa International Hospital, Islamabad (IRB no. 009829-2020). A non-probability convenience sampling technique was used to enroll patients. The sample size was calculated using OpenEpi. By keeping the incidence of WIPO as $27.4 \%, 11,12$ confidence level of $85 \%$, and a margin of error as $10 \%$, a sample size of 42 was determined.

Inclusion Criteria: Patients of both genders, on invasive mechanical ventilation, meeting the criteria for weaning and undergoing a planned SBT with pressure support $5-8 \mathrm{~cm}$ of water, were included in the study.

Exclusion Criteria: Patients below the age 18 years, those with underlying malignancy or those with poor cardiac echogenicity (mitral inflow can not be determined) were excluded from the study.

SBT was performed on a $\mathrm{T}$ piece or P-support of $5-8 \mathrm{~cm}$ of $\mathrm{H}^{2} \mathrm{O}$. The duration of the SBT was at least 30 minutes or above. Those who completed the SBT successfully were extubated but followed for the next 48 hours. Treating intensivists had the authority to stop SBT if not tolerated. The diagnosis of weaning failure was made if SBT was stopped due to poor tolerance or patient required reintubation within 48 hours of extubating. Certain variables which included clinical as well as biological variables were noted before an SBT and after 30 minutes (sooner if SBT failed). Respiratory rate, heart rate, oxygen saturation and blood pressure were the clinical variables while arterial blood gas analysis was recorded as a biological variable. Variables of Echocardiography were recorded at the time of admission only to see baseline diastolic dysfunction and left ventricular ejection fraction.

Lung ultrasound was performed before and after an SBT, using the Mindray ultrasound device model 3C-5P 3.5 HZ convex probe. The primary investigator saved the images of the lung ultrasound. The analysis of lung ultrasound was based on BLUE protocol. According to the protocol, B-lines were counted in the short axis view of the ribs between the ribs, at standardized four points of BLUE protocol. In case of pneumonia, the counting of B-lines was made at the nearest alternative points (flexible BLUE points). Probe was applied to the same specific area. The duration of each test was less than five minutes. Images were saved and the number of B-lines and difference in number of B-lines (Delta-B-lines) were counted. Reference diagnosis of WIPO was made by an intensivist expert in lung ultrasound. The intensivist was not informed of the objectives of the study to prevent bias.

The data analysis was performed using the statistical package for social sciences (SPSS) version 26. The study variables were presented using frequency and mean with standard deviation. To determine the association of WIPO and the number of Delta-B-lines on lung ultrasonography, chi square test was applied. The $p$-value of $\leq 0.05$ was kept as cut off for statistical significance.

\section{RESULTS}

Forty-two study participants who fulfilled the eligibility criteria were included in the study. There were $23(54.8 \%)$ male and $19(45.2 \%)$ female patients. The majority of the patients were between the ages of sixty and eighty years. Echocardiography was done for evaluation of baseline cardiac function in all patients. Echocardiography results showed no diastolic dysfunction in $33(78.6 \%)$ patients while grade 1-2 diastolic dysfunction was present in 9 patients $(21.4 \%)$. Systolic ejection fraction was more than $50 \%$ in 32 patients, 30 $50 \%$ in 8 patients and less than $30 \%$ in 2 patients. SBT duration was 30 minutes in 37 (88.1\%) patients while in 
five $(11.9 \%)$ patients the duration for SBT was greater than thirty minutes (Table-I).

\begin{tabular}{|c|c|}
\hline Characteristics & n (\%) \\
\hline \multicolumn{2}{|l|}{ Gender } \\
\hline Male & $23(54.8 \%)$ \\
\hline Female & $19(45.2 \%)$ \\
\hline \multicolumn{2}{|l|}{ Age Groups } \\
\hline 20-39 years & $7(16.7 \%)$ \\
\hline $40-59$ years & $8(19.0 \%)$ \\
\hline $60-80$ years & $25(59.5 \%)$ \\
\hline above 80 years & $2(4.8 \%)$ \\
\hline \multicolumn{2}{|l|}{ Diastolic Dysfunction } \\
\hline Not present & $33(78.6 \%)$ \\
\hline Grade 1 or 2 & $9(21.4 \%)$ \\
\hline \multicolumn{2}{|l|}{ Ejection Fraction } \\
\hline More than $50 \%$ & $32(76.2 \%)$ \\
\hline $30-50 \%$ & $8(19.0 \%)$ \\
\hline Less than $30 \%$ & $2(4.8 \%)$ \\
\hline \multicolumn{2}{|c|}{ Spontaneous Breathing Trial (SBT) Duration } \\
\hline 30 minutes & $37(88.1 \%)$ \\
\hline Greater than 30 minutes & $5(11.9 \%)$ \\
\hline \multicolumn{2}{|c|}{ Spontaneous Breathing Trial (SBT) Outcome } \\
\hline Successful Extubation & $28(66.7 \%)$ \\
\hline Unsuccessful Extubation & $14(33.3 \%)$ \\
\hline
\end{tabular}

Seven $(16.7 \%)$ patients were confirmed diagnosis of WIPO while 35 (83.3\%) were not confirmed diagnosis of WIPO as shown in Figure.

Frequency of Confirmed Diagnosis of Weaning Induced Pulmonary Edema (WIPO)

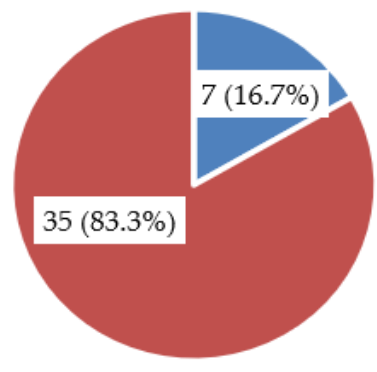

- Confirmed diagnosis of WIPO

- Not confirmed diagnosis of WIPO

Figure: Frequency of confirmed diagnosis of weaning induced pulmonary edema (WIPO).

In $14(33.3 \%)$ patients, there was SBT failure. WIPO reference diagnosis was made in $7(50 \%)$ cases out of 14 cases. Delta-B-lines $\geq 6$ diagnosed WIPO with $100 \%$ accuracy. In the WIPO patients, the increase in the number of B-lines to $\geq 6$ was noted in 7 cases with reference diagnosis of WIPO. The number of Delta-Blines significantly correlated with the occurrence of WIPO in patients $(p<0.001)$ shown in Table-II.
Among the rest of seven patients who failed SBT but without reference diagnosis of WIPO, the Delta-Blines were six (6) in four patients. WIPO was diagnosed with this profile in the 14 cases who failed SBT with a sensitivity of $100 \%$ and a specificity of $77.78 \%$.

Table-II: The association of weaning induced pulmonary edema and number of delta $B$ lines on lung ultrasonography.

\begin{tabular}{|c|c|c|c|c|c|c|c|c|}
\hline \multirow{2}{*}{$\begin{array}{l}\text { WIP0 } \\
\text { Reference } \\
\text { Diagnosis }\end{array}$} & \multicolumn{7}{|c|}{ Number of Delta B Lines (\%) } & \multirow{2}{*}{$\begin{array}{c}p- \\
\text { value }\end{array}$} \\
\hline & 0 & 1 & 2 & 3 & 4 & 5 & 6 & \\
\hline Confirmed & - & - & - & - & - & - & $\begin{array}{c}7 \\
(100)\end{array}$ & \multirow{2}{*}{$<0.001$} \\
\hline $\begin{array}{l}\text { Not } \\
\text { Confirmed }\end{array}$ & \begin{tabular}{|c|}
3 \\
$(100)$
\end{tabular} & \begin{tabular}{|c|}
14 \\
$(100)$
\end{tabular} & $\begin{array}{c}13 \\
(100)\end{array}$ & $\begin{array}{c}2 \\
(100)\end{array}$ & $\begin{array}{c}3 \\
(100)\end{array}$ & - & 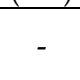 & \\
\hline
\end{tabular}

\section{DISCUSSION}

Elevation in pulmonary artery wedge pressure (PAWP) after a SBT remains the standard diagnostic criterion for detection of WIPO. Changes in PAWP are measured through pulmonary artery catheter (PAC). 3,6,11 This technique is invasive and associated with complications such as arrhythmias, pulmonary infarction, catheter knotting and catheter entanglement with other endovascular structures. Therefore, more recently, less invasive alternative methods for the diagnosis of WIPO are being explored.

The remarkable rise in BNP or the NT-BNP levels during a weaning trial is one of the indirect but reliable methods to detect WIPO.5,12 Another biological technique to detect WIPO depends on hemoconcentration induced during pulmonary edema resulting from the passage of a significant amount of plasma throughout the lung alveolar-capillary interface. Increase in hemoconcentration, measured through elevation in hemoglobin and/or plasma proteins helps in diagnosing WIPO with certainty. ${ }^{13}$ Echocardiography can reliably measure left ventricular filling pressure via Doppler flow study of mitral inflow and annulus motion. During WIPO, the increase in lung water outside the vascular space is directly detected by thermodilution technique that is transpulmonary. ${ }^{14}$

Lung ultrasound is a promising modality in being noninvasive and quick. It detects interstitial syndrome in the lungs that is the first stage of hemodynamic pulmonary edema. The interstitial syndrome is diffuse so it can be detected easily at the anterior chest wall points thus detecting WIPO at an earlier stage. ${ }^{15,16}$

The current study indicates that lung ultrasonography can accurately detect WIPO. Delta-B-lines of equal to or more than six established the diagnosis of WIPO. WIPO developed in half of the patients who 
failed SBT. In 2019, Frere et al revealed that out of the 33 cases that failed SBT, ${ }^{17}$ had WIPO. Among those patients, $15 / 17$ had B lines of $\geq 6$. The authors concluded that Delta-B-lines $\geq 6$ on four anterior points permitted the diagnosis of WIPO. ${ }^{12}$ Half of the population, who had SBT failure, also had WIPO, highlighting WIPO as the most common cause of weaning failure. Our study findings are supported by previous studies. 17-19

In a recent study by Bouhemad et al it was found that lung ultrasound score was significantly associated with risk of extubation failure $(p=0.008) .{ }^{20}$ Yamaga et al questioned the reliability of lung ultrasound for diagnosing WIPO. The author claimed that since several B-lines could move quickly with lung sliding signs because of tachypnoea, it might cause large variation in the number of B-lines, as per intensivist. Therefore, even though the ultrasonography is less invasive, the reliability of the technique still needs further investigation. ${ }^{21}$ As we know failure to wean is associated with poor prognosis, detecting WIPO with reliability prompts the initiation of its treatment, which results in better results. $22-23$

The current study indicated that weaning induced pulmonary edema is a common occurrence in the critically ill patients in our setting. Lung ultrasound is a non-invasive tool, which can accurately detect development of WIPO in patients with the help of Delta-Blines. Prompt diagnosis and emergency treatment can improve patient outcomes. Further large-scale multicenter studies can ascertain these findings more accurately.

\section{CONCLUSION}

The study indicates that Delta-B-lines $\geq 6$ diagnosed the weaning induced pulmonary edema with the best accuracy.

\section{Conflict of Interest: None.}

\section{Authors' Contribution}

GR: Study conception, study design, draft writing, ZS: Data acquisition, review editing, TM: Literature review, study design, NK: Critical appraisal \& revision, MR: Data acquisition \& result interpretation, AA: Data acquisition \& result interpretation KA: Draft writing.

\section{REFERENCES}

1. Fernandez MM, González-Castro A, Magret M, Bouza MT. Reconnection to mechanical ventilation for $1 \mathrm{~h}$ after a successful spontaneous breathing trial reduces reintubation in critically ill patients: a multicenter randomized controlled trial. Intensive Care Med 2017; 43(11): 1660-1667.

2. Windisch W, Dellweg D, Geiseler J, Westhoff M, Pfeifer M, Suchi S, et al. Prolonged weaning from mechanical ventilation: results from specialized weaning centers - a registry-based study from the Wean Net Initiative. Dtsch Ärztebl Int 2020; 117(12): 197-200.
3. Jung YT, Kim MJ, Lee JG, Lee SH. Predictors of early weaning failure from mechanical ventilation in critically ill patients after emergency gastrointestinal surgery: a retrospective study. Med (Baltimore) 2018; 97(40): e12741.

4. Dres M, Teboul JL, Monnet X. Weaning the cardiac patient from mechanical ventilation. Curr Opin Crit Care 2014; 20(5): 493-498.

5. Vignon P. Cardiovascular failure and weaning. Ann Transl Med 2018; 6(18): 354.

6. Routsi C, Stanopoulos I, Kokkoris S, Sideris A, Zakynthinos S. Weaning failure of cardiovascular origin: how to suspect, detect and treat-a review of the literature. Ann Intensive Care 2019; 9(1): 6-8.

7. Price S, Platz E, Cullen L, Tavazzi G, Christ M, Cowie MR, et al. Expert consensus document: echocardiography and lung ultra-sonography for the assessment and management of acute heart failure. Nat Rev Cardiol 2017; 14(7): 427-440.

8. Dres M, Teboul JL, Anguel N, Guerin L, Richard C, Monnet X Extravascular lung water, B-type natriuretic peptide, and blood volume contraction enable diagnosis of weaning-induced pulmo-nary edema. Crit Care Med 2014; 42(8): 1882-1889.

9. Pičuljan A, Protić A, Haznadar M, Šustić A. the role of b-line artifacts on lung ultrasound in critically Ill patients. Signa Vitae 2020; 16(2): 1-7.

10. Sartini S, Frizzi J, Borselli M, Sarcoli E, Granai C, Gialli V, et al. Which method is best for an early accurate diagnosis of acute heart failure? Comparison between lung ultrasound, chest x-ray and NT proBNP performance: a prospective study. Intern Emerg Med 2017; 12(6): 861869.

11. El Hadidy S, Saad M, El Hossany R, El Gohary T, El Ghobashy M Coinciding changes in $\mathrm{B}$ lines patterns, haemoglobin and hematocrit values can predict outcomes of weaning from mecha-nical ventilation. Open Access Maced J Med Sci 2019; 7(23): 4010.

12. Ferré A, Guillot M, Lichtenstein D, Mezière G, Richard C, Teboul JL, et al. Lung ultrasound allows the diagnosis of weaning-indu-ced pulmonary oedema. Intensive Care Med 2019; 45(5): 601-608.

13. Jindal SK. Textbook of pulmonary and critical care medicine: Two-Volume Set. JP Medical Ltd; 2017, Available at: https://www.amazon.com/ Textbook-Pulmonary-Critical-Care-Medicine/dp/9385999990

14. Haas SA, Trepte CJ, Nitzschke R, Jürgens TP, Goepfert MS, Goetz AE, et al. An assessment of global end-diastolic volume and extravascular lung water index during one-lung ventilation: is transpulmonary thermodilution usable? Anesth Analg 2013; 117(1): 83-90.

15. Lichtenstein DA, Meziere GA. Relevance of lung ultrasound in the diagnosis of acute respiratory failure: the BLUE protocol. Chest 2008; 134(1): 117-125.

16. Lichtenstein DA, Mezière GA. The BLUE-points: three standar-dized points used in the BLUE-protocol for ultrasound assess-ment of the lung in acute respiratory failure. Crit Ultrasound J 2011; 3(2): 109-110.

17. Dres M, Teboul JL, Anguel N, Guerin L, Richard C, Monnet X. Extravascular lung water, B-type natriuretic peptide, and blood volume contraction enable diagnosis of weaning-induced pulmonary edema. Crit Care Med 2014; 42(8): 1882-1889.

18. Dres M, Teboul JL, Anguel N, Guerin L, Richard C, Monnet X. Passive leg raising performed before a spontaneous breathing trial predicts weaning-induced cardiac dysfunction. Intensive Care Med 2015; 41(3): 487-494.

19. Teboul JL, Monnet X, Richard C. Weaning failure of cardiac origin: recent advances. In Yearbook of Intensive Care and Emergency Medicine Springer, Berlin, Heidelberg, 2010 Crit Care 2010; 14(2): 175-182.

20. Bouhemad B, Mojoli F, Nowobilski N, Hussain A, Rouquette I, Guinot PG, et al. Use of combined cardiac and lung ultrasound to predict weaning failure in elderly, high-risk cardiac patients: a pilot study. Intensive Care Med 2020; 46(3): 475-484.

21. Yamaga S, Ohshimo S, Shime N. Is lung ultrasonography really useful for diagnosing weaning-induced pulmonary oedema?. Intensive Care Med 2019; 45(9): 1329-1330.

22. Thille AW, Richard JC, Brochard L. The decision to extubate in the intensive care unit. Am J Respir Crit Care Med 2013; 187(12): 12941302.

23. Sanfilippo F, Di-Falco D, Noto A, Santonocito C, Morelli A, Bignami $\mathrm{E}$, et al. Association of weaning failure from mechanical ventilation with transthoracic echocardiography parameters: a systematic review and meta-analysis. Br J Anaesth 2021; 126(1): 319-330. 